\title{
Phlebologische Funktionsdiagnostik: Praktische Anwendung
}

\author{
Teil 1: Die digitale Photoplethysmografie
}

\section{Venous Functional Diagnostics: Hands-On Approach}

\author{
Part 1: Digital Photoplethysmography
}

\section{Autoren}

S. Kahl, G. Bruning, J. Woitalla-Bruning

\section{Institute}

Krankenhaus Tabea GmbH \& Co. KG, Zentrum für Venen- und Dermatochirurgie, Hamburg

\section{Schlüsselwörter}

Venöse Funktionsdiagnostik, digitale Photoplethysmografie, venöse Wiederauffüllzeit

Key words

Venous functional diagnostics, digital photoplethysmography, venous refilling time

eingereicht 24.02.2019

akzeptiert 26.02.2019

Bibliografie

DOI https://doi.org/10.1055/a-0863-9365

Online Publikation: 24.04.2019

Phlebologie 2019; 48: 187-191

(c) Georg Thieme Verlag KG Stuttgart · New York

ISSN 0939-978X

Korrespondenzadresse

Dr. med. Sebastian Kahl

Facharzt im Zentrum für Venen- und Dermatochirurgie

Krankenhaus Tabea GmbH \& Co. KG

Kösterbergstraße 32

22587 Hamburg

Tel.: 04086692200

E-Mail: SKahl@tabea-krankenhaus.de

\section{ZUSAMMENFASSUNG}

Trotz der Dominanz der farbkodierten Duplexsonografie in der Diagnostik der Phlebologie gibt es weiterhin Fragestellungen, bei denen klassische Verfahren der phlebologischen Funktionsdiagnostik eine wichtige Rolle bei der Therapieentscheidung spielen. Während die farbkodierte Duplexsonografie detaillierten Aufschluss über die Kaliber und die Refluxsituation des Venensystems liefern kann, ist sie jedoch nicht in der Lage, eine abschließende Aussage zur Hämodynamik zu treffen. Hierzu dient die venöse Funktionsdiagnostik und als Referenzverfahren insbesondere die invasive Phlebodynamometrie (PDM). Die PDM ist die einzige Methode zur direkten Messung der ambulatorischen venösen Hypertonie und hat daher eine große Bedeutung im Rahmen der funktionellen phlebologischen Diagnostik, insbesondere beim postthrombostischen Syndrom (PTS). Sie kann am verlässlichsten darüber Auskunft geben, ob es sich bei Vorliegen einer Varikosis mit kurzem Reflux im Rahmen eines PTS um eine besserbare Varikosis handelt oder nicht.

Das Ziel dieses dreiteiligen Artikels besteht darin, die praktische Anwendung der klassischen Verfahren zur phlebologischen Funktionsdiagnostik im klinischen Rahmen zu demonstrieren und die Aussagekraft der entsprechend erhobenen Daten zu erläutern. Die praktische Anwendung der digitalen Photoplethysmografie (D-PPG), der PDM und der Venenverschlussplethysmografie (VVP) ist prinzipiell nicht schwer. Für die Durchführung wird jedoch geschultes Personal benötigt, um Anwendungsund Messfehler zu vermeiden. In diesem ersten Teil soll die digitale Photoplethysmografie vorgestellt und erläutert werden.

\section{ABSTRACT}

Duplex ultrasound (DUS) is currently the most important method concerning the assessment of venous disease. In many cases it has replaced conventional methods that where designed for venous functional diagnostics. But still, there are some medical questions that can only be answered by data collected in connection with classic tests of venous functional diagnostics. For example, DUS can give you detailed information about venous diameter and venous reflux. But it cannot give you adequate information about venous pressure. So when it comes to questions concerning venous pressure, you depend on venous functional diagnostics. Within this context, direct venous pressure measurement (DVPM) is the most important and reliable method to find out if a patient would profit from surgical therapy of varicose veins (especially if your patient suffers from post-thrombotic syndrome).

The aim of this three-part article is to demonstrate the practical use of all classic methods of venous functional diagnostics in a clinical setting and to point out what conclusions can be made based on collected data. In this first part, we present digital photoplethysmography (D-PPG). D-PPG, DVPM and venous occlusion plethysmopraphy (VOP) can be performed easily by trained medical staff and despite the dominance of DUS, these classic methods still play a role in the assessment of venous disease. 


\section{Einleitung}

Mit Einführung der farbkodierten Duplexsonografie in die phlebologische Diagnostik sind die klassischen Verfahren der venösen Funktionsdiagnostik im Alltag etwas in den Hintergrund getreten $[1,2]$. Bezüglich ihrer Anwendung mag somit der eine oder andere jüngere Kollege auf dem Gebiet der Phlebologie nur wenig praktische Erfahrung aufweisen.

Wir möchten mit diesem dreiteiligen Artikel daher die Anwendung der entsprechenden phlebologischen Untersuchungsmethoden mit Hilfe von anschaulichen Bildern aus dem klinischen Alltag beschreiben. In diesem ersten Teil wird die Digitale Photoplethysmografie (D-PPG) vorgestellt. In den beiden folgenden Ausgaben dieses Journals werden wir uns dann der Phlebodynamometrie (PDM) und zuletzt auch der Venenverschlussplethysmografie (VVP) widmen.

Für die genannten Verfahren gibt es mobile Komplett-Systeme inkl. verschiedener Mess-Sonden und Sensoren, zentraler RechenEinheit, Display, Drucker und Manschetten-Halterung ( $\mathbf{A b b} \mathbf{1}$ ).

\section{Digitale Photoplethysmografie (D-PPG)}

\section{Untersuchungsablauf}

Die D-PPG wird am sitzenden Patienten durchgeführt. Die Knie sollen dabei in einem Winkel von ca. 110 Grad gebeugt sein. Der LichtSensor ( $>$ Abb. 2 ) wird ca. $10 \mathrm{~cm}$ proximal vom Innenknöchel des zu untersuchenden Beines auf die gesunde (nicht trophisch gestörte) Haut geklebt ( $\mathbf{A b b}$. 3) [2, 3].

Dann führt der sitzende Patient im Sprunggelenk wiederholt ca. 8-10 Plantarflexionen mit anschließender Rückkehr in die Ruheposition durch. Durch die aktivierte Muskel-Waden-Pumpe wird dann das Blut aus dem Unterschenkel über das venöse Gefäßsystem zurück zum Herzen gepumpt ( $>$ Abb. 4). Im nächsten Schritt wird der Zustand nach einer operativen Entfernung eines potentiell auszuschaltenden Gefäßes simuliert. Hierzu schaltet man das varikös veränderte Gefäß durch einen Stauschlauch bzw. durch ein Tourniquet funktionell aus ( $>$ Abb.5). Anschließend wiederholt man die Untersuchung mit dem zuvor beschriebenen Manöver [2, 3].

\section{Funktionsprinzip}

Die D-PPG ist ein digitales Verfahren aus der Gruppe der sog. photoplethysmografischen Verfahren zur phlebologischen Funktionsdiagnostik. Zu dieser Gruppe zählen auch die Photoplethysmografie (PPG) und die Lichtreflexionsrheografie (LRR) nach Blazek/Wienert. Allen Verfahren ist gemeinsam, dass sie Licht im Infrarot-Bereich einsetzen [2]. In der täglichen Anwendung nimmt heute die D-PPG von allen 3 Verfahren den wichtigsten Platz ein.

Bei der D-PPG wird das Infrarotlicht über einen auf der Haut klebenden Licht-Sensor abgeben. Anschließend kann man über den Sensor den entsprechenden Reflexionsgrad (Menge des reflektierten Infrarotlichts) bestimmen. Da Hämoglobin Licht absorbiert, ist es auf diese Weise möglich, indirekt auf die Blutfüllung des untersuchten Areals bzw. auf die Blutfüllung des subkutanen Venenplexus zu schließen [2].

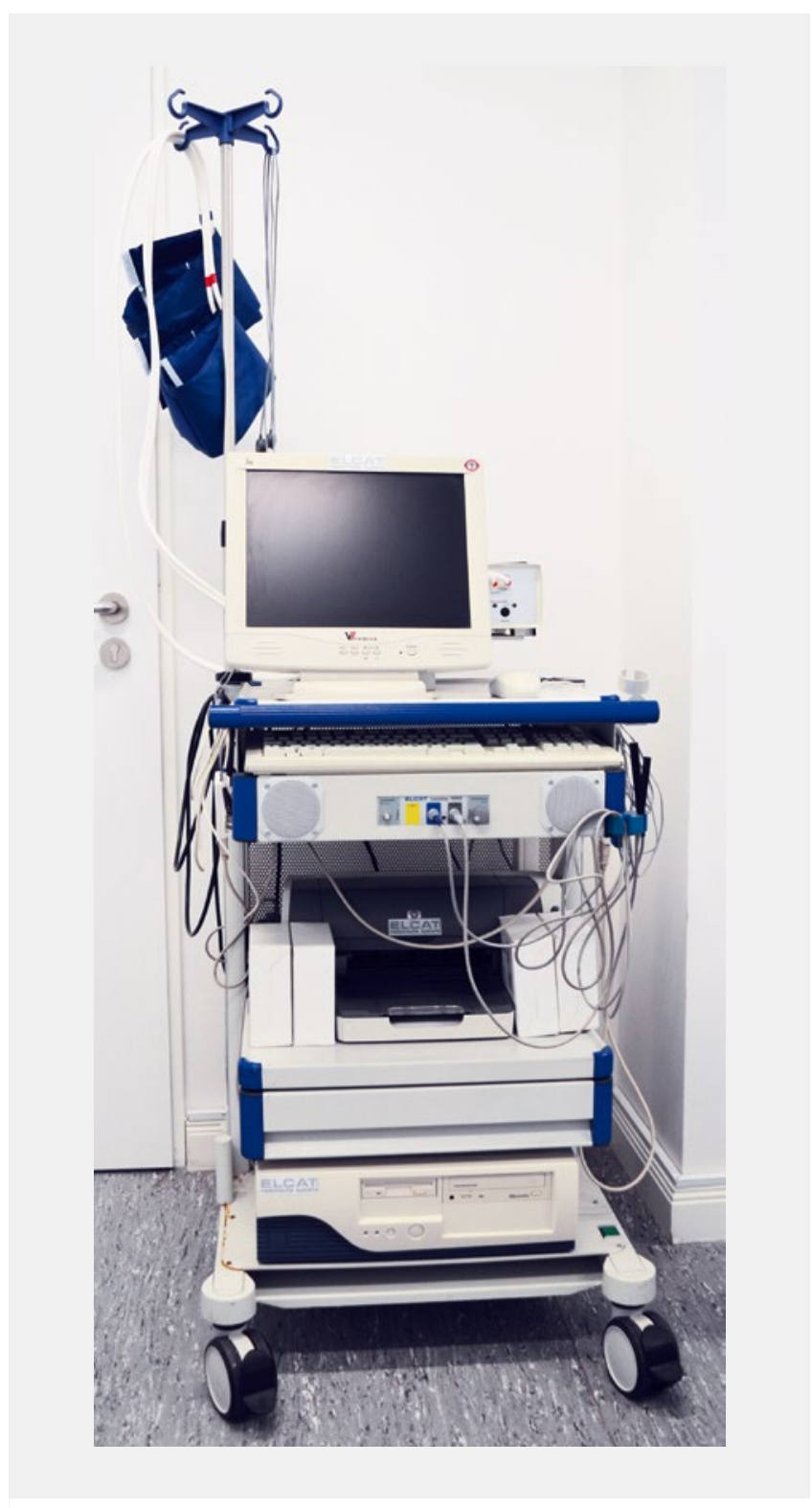

-Abb. 1 Mobiles Komplett-System inkl. verschiedener Mess-Sonden und Sensoren, zentraler Rechen-Einheit, Display, Drucker und Manschetten-Halterung.

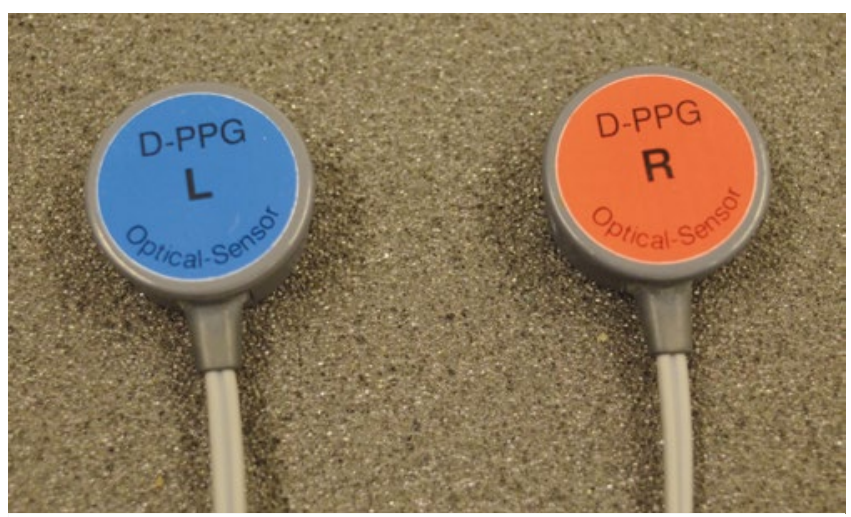

Abb. 2 Rückseite der Licht-Sensoren für das rechte Bein (rot) und das linke Bein (blau). 


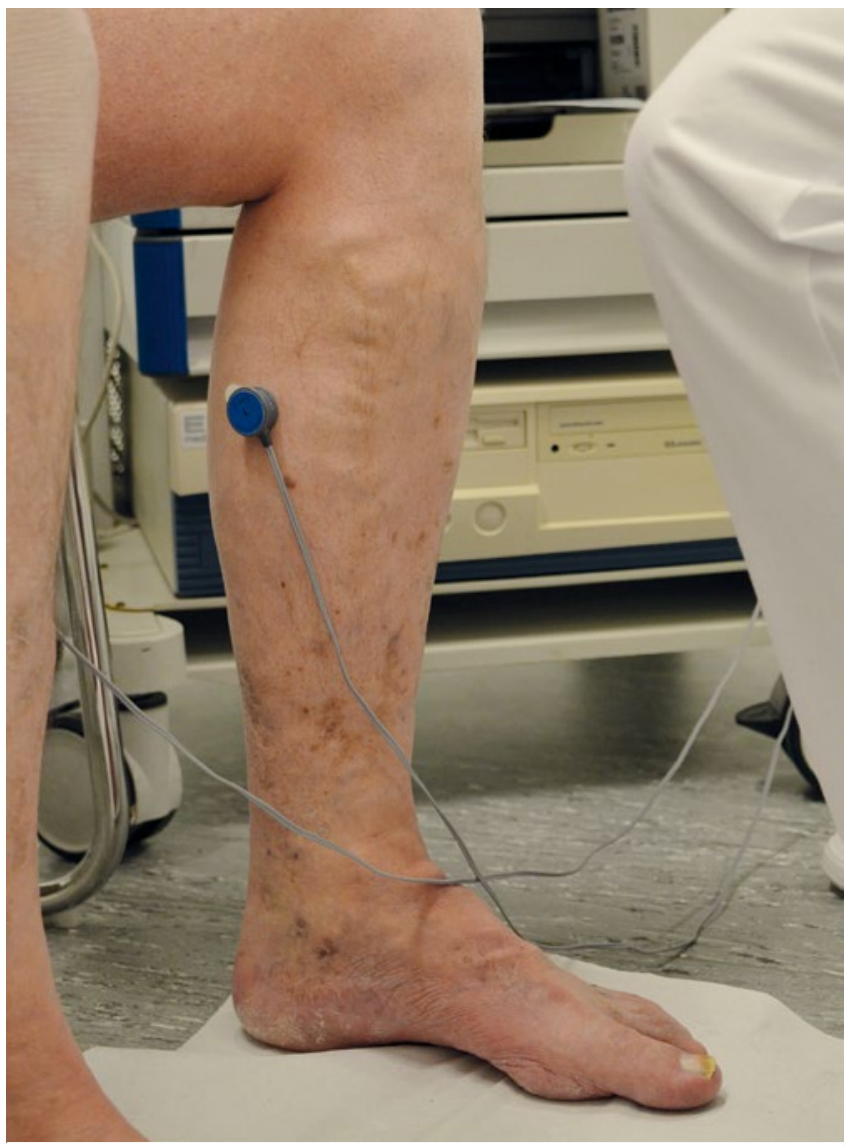

- Abb. 3 Demonstration des Lichtsensors am zu untersuchenden Bein, hier weiter proximal als $10 \mathrm{~cm}$ vom Innenknöchel aufgebracht aufgrund von trophischen Störungen der Haut.

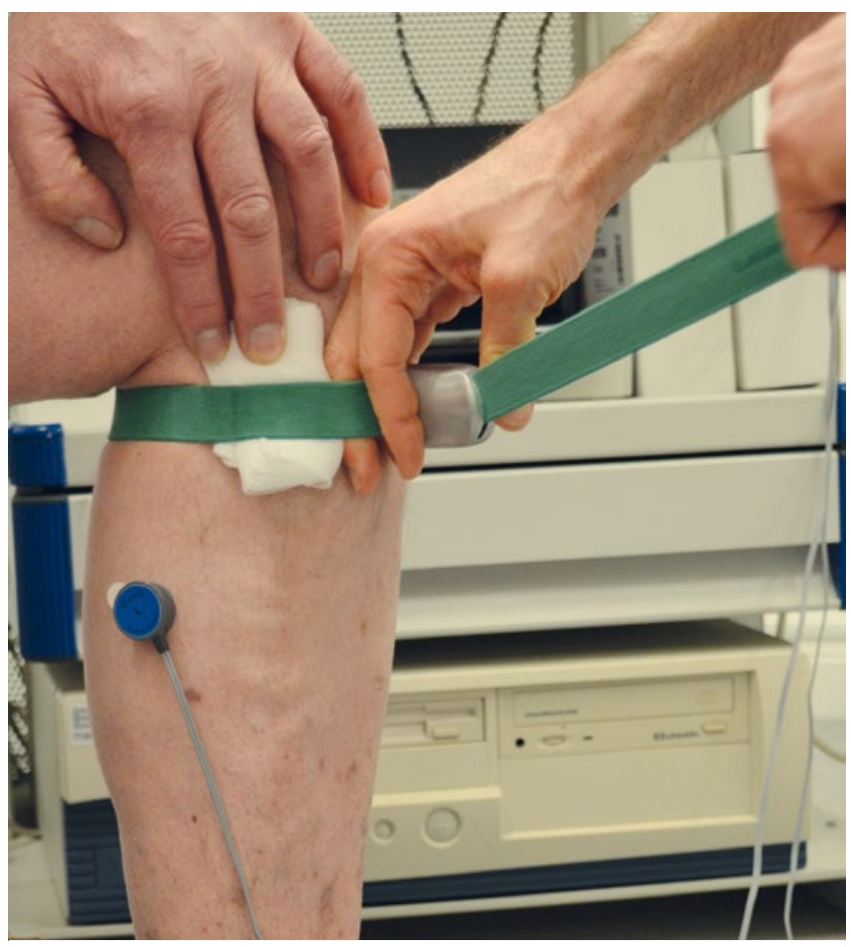

- Abb. 5 Simulation des Zustandes nach operativer Entfernung des varikösen Gefäßes durch Anlegen eines Stauschlauches.

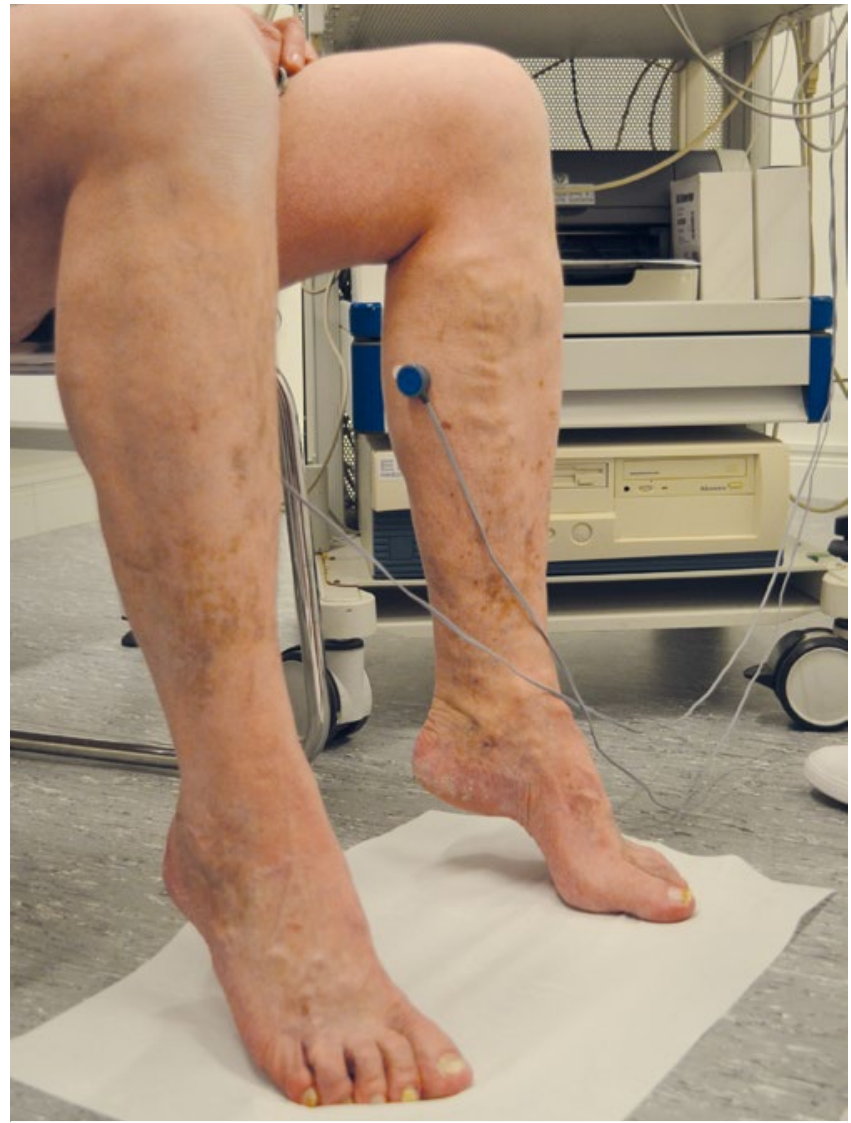

Abb. 4 Durch wiederholte Plantarflexion und Aktivierung der Muskelwadenpumpe wird das Blut aus dem venösen Gefäßsystem des Beines zurück Richtung Herz gepumpt.

\section{Indikationen}

Die D-PPG wird vor allem bei der Frage eingesetzt, ob der venöse Abstrom aus einem Bein durch die operative Entfernung von varikösen Gefäßen gebessert werden kann oder nicht, ob also eine „besserbare“ venöse Insuffizienz vorliegt (z. B. bei Leitveneninsuffizienz oder postthrombotischem Syndrom) [2-4].

\section{Messparameter und Interpretation}

Die $>$ Abb. 6 zeigt die primären Parameter, die bei der D-PPG dargestellt werden.

Auf der x-Achse ist der zeitliche Verlauf dargestellt, auf der $y$-Achse der Anteil des reflektierten Lichtes. Je größer der Wert auf der y-Achse, desto höher der Anteil des reflektierten Lichtes und desto geringer die Füllung des untersuchten subkutanen Venenplexus mit Blut.

Anhand der dargestellten Parameter kann abgelesen werden, wie lange es dauert, bis nach einem Manöver zur Entleerung des subkutanen Venenplexus wieder ein kontinuierlicher Füllungszustand erreicht ist. Das entsprechende Zeitintervall wird als Venöse Wiederauffüllzeit (t0; Normwert: > 25 Sekunden) bezeichnet und stellt die entscheidende Variable dar, die bei der D-PPG berechnet wird [2, 3]. Ist die venöse Wiederauffüllzeit verkürzt, so liegt eine venöse Funktionsstörung vor. Diese teilt man je nach Betrag des gemessenen Wertes in die Grade I bis III ein: 


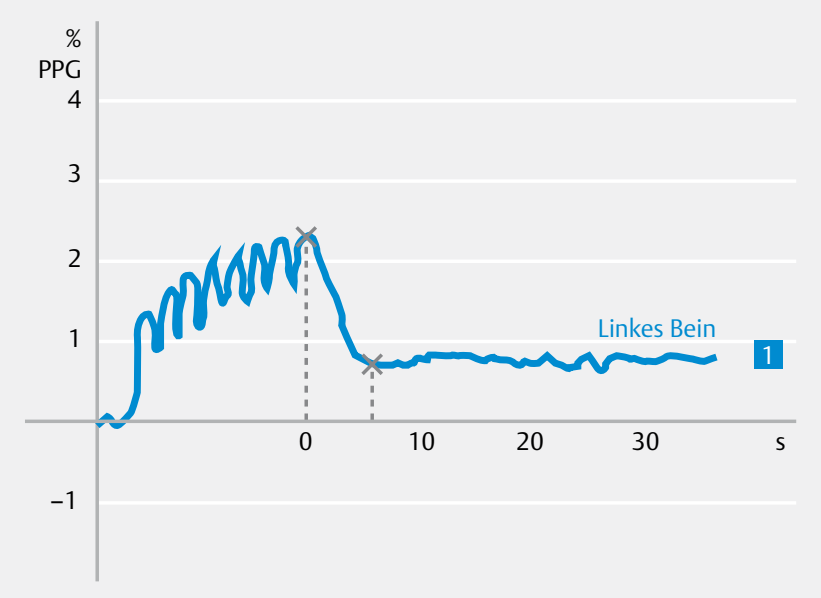

-Abb. 6 Beispiel für Untersuchungsbefund der D-PPG ohne anliegenden Stauschlauch.

- Grad 1: Venöse Wiederauffüllzeit t0 zwischen 20 und 25 Sekunden

- Grad 2: Venöse Wiederauffüllzeit t0 zwischen 10 und 19 Sekunden

- Grad 3: Venöse Wiederauffüllzeit t0 unter 10 Sekunden

Je kürzer also die Zeit bis zur Wiederauffüllung des subkutanen Venenplexus, desto stärker ist die Ausprägung der venösen Funktionsstörung [2, 3].

- Abb. 7 zeigt Messergebnisse desselben Patienten wie in - Abb. 6, in diesem Fall jedoch nach Ausschaltung eines insuffizienten Gefäßes mit Hilfe eines Stauschlauches. Das Zeitintervall zwischen dem relativen Maximum der Kurve bei 0 Sekunden und Erreichen des Plateaus bei etwa 17 Sekunden ( $>$ Abb. 7) ist im Vergleich zum Manöver davor ( Abb. 6) deutlich vergrößert. Der Patient würde somit von der operativen Entfernung des varikösen Gefäßes am getesteten Bein profitieren. Es liegt also eine „besserbare" Varikosis vor. [4].

\section{Potentielle Fehlerquellen}

Beim Kleben der Sensoren muss darauf geachtet werden, dass diese nur auf intakte Haut ohne trophische Störungen aufgebracht werden. Die Sensoren dürfen also nicht über einem Hautareal angebracht werden, dass z. B. entsprechend einer Stauungsdermatitis, einer Purpura jaune d'ocre, einer Dermatosklerose oder eines Ulkus cruris verändert ist. Hier besteht die Gefahr, dass das Infrarotlicht bei der Untersuchung nicht in ausreichendem Maße bis zum subkutanen Venenplexus durch die Haut dringt [2, 3]. Die Folge wären Messfehler. Aufgrund dieser Überlegungen wurden die Sensoren bei dem Patienten in unserem Beispiel etwas weiter proximal aufgebracht als gewöhnlich.

Die angesprochene Anwendungseinschränkung limitiert das Verfahren der D-PPG bei entscheidenden Fragen zur Operationsindikation bei Varikosis bzw. postthrombotischem Syndrom. Denn gerade Patienten mit Varikosis weisen oft throphische Störungen der Haut auf. Bei ausgeprägten bzw. großflächigen Hautveränderungen steht alternativ die PDM zur Verfügung. Weiterhin darf der

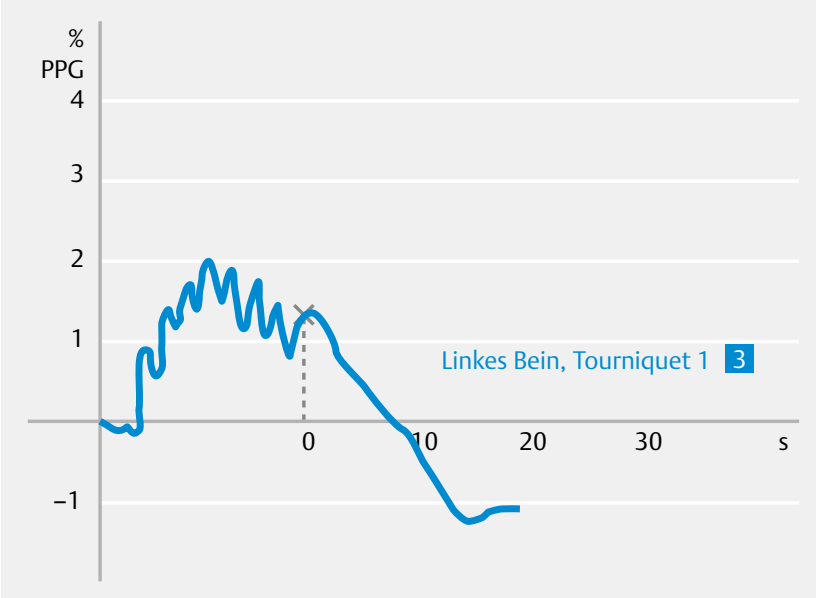

Abb. 7 Beispiel für Untersuchungsbefund der D-PPG bei anliegendem Stauschlauch.

Lichtsensor bei der D-PPG nicht direkt über einer Perforans-Vene oder einem Varixknoten angebracht werden, da das Blut einer solchen Vene bzw. eines solchen Knotens das Messergebnis ebenfalls verfälschen würde [2].

Es ist zu beachten, dass das Verfahren standardisiert ist für eine Zimmertemperatur von ca. $20^{\circ}$ Celsius, so dass eine Hauttemperatur des Patienten von $20-24^{\circ} \mathrm{C}$ vorliegt.

\section{FAZIT}

Die praktische Anwendung der D-PPG ist prinzipiell nicht schwer. Für die Durchführung wird jedoch geschultes Personal benötigt, um Anwendungs- und Messfehler zu vermeiden.

Trotz der Dominanz der farbkodierten Duplexsonografie in der Diagnostik der Phlebologie gibt es weiterhin einige Fragestellungen, bei denen klassische Verfahren der phlebologischen Funktionsdiagnostik eine wichtige Rolle bei der Therapieentscheidung spielen [1-3, 5, 6]. Denn während die farbkodierten Duplexsonografie detaillierten Aufschluss über die Gefäßkaliber und die Refluxsituation des Venensystems liefert, ist sie jedoch nicht in der Lage, eine abschließende Aussage zur Hämodynamik zu treffen. Hierzu dient die venöse Funktionsdiagnostik, zu der die D-PPG zählt. Sie kann Auskunft darüber geben, ob ein Patient von einem Varizenausschaltenden Verfahren profitieren würde. Letztere Information kann mit Hilfe der Duplexsonografie nicht gewonnen werden. 


\section{Interessenkonflikte}

S. Kahl: Der Autor gibt an, dass keine Interessenkonflikte bestehen.

G. Bruning: Reisekostenübernahme im Rahmen von Kongressbesuch durch die Firma Bauerfeind AG.

J. Woitalla-Bruning: Reisekostenübernahme im Rahmen von Kongressbesuch durch die Firma Bauerfeind AG. Vortragshonorar von der SIGVARIS GmbH.

\section{Literatur}

[1] Mühlberger D, Reich-Schupke S, Mumme A et al. Funktionsdiagnostik in der Phlebologie. vasomed, 30. Jahrgang_6_2018; S.283-287.

[2] Rabe E, Gerlach HE. Praktische Phlebologie. Empfehlung zur differenzierten Diagnostik und Therapie phlebologischer Krankheitsbilder.
2. vollständig überarbeitete Auflage. Georg Thieme Verlag, Stuttgart, New York, 2006.

[3] Pannier F, Gerlach H, Stücker et al. AWMF-Leitlinien-Register -Nr. 037/008 Entwicklungsstufe: 1 Zuletzt aktualisiert: Mai 2012 Leitlinie: Venöse Diagnostik mit der Licht-Reflexions-Rheographie/Photoplethysmographie.

[4] Hach W, Gruss JD, Hach-Wunderle et al. VenenChirurgie: Leitfaden für Gefäßchirurgen, Angiologen, Dermatologen und Phlebologen. 2. Auflage; Schattauer, 2007.

[5] Gerlach HE, Rabe E, Stücker M et al. AWMF-Leitlinien-Register -Nr. 037/014 Entwicklungsstufe: 1 Zuletzt aktualisiert: Mai 2012 Leitlinie: Venöse Diagnostik mit der Venenverschlussplethysmographie mittels Dehnungsmessstreifen.

[6] Rabe E, Hartmann K, Gerlach H et al. Schimmelpfennig L, Pannier F. AWMF-Leitlinien-Register-Nr. 037/013 Entwicklungsstufe: 1 Zuletzt aktualisiert: Mai 2012 Leitlinie: Venöse Diagnostik mit der Phlebodynamometrie. 